\title{
QUI VIENT À NUIT DEBOUT ? PARIS, PLACE DE LA RÉPUBLIQUE, AVRIL- JUIN 2016 TROIS MÉTHODES POUR UNE QUESTION
}

\author{
Stéphane Baciocchi, Alexandra Bidet, Pierre Blavier, Carole Gayet-Viaud, Erwan Le \\ Méner
}

Presses Universitaires de France | « Sociologie »

2020/3 Vol. 11 | pages 251 à 266

ISSN 2108-8845

ISBN 9782130823780

Article disponible en ligne à l'adresse :

https://www.cairn.info/revue-sociologie-2020-3-page-251.htm

Distribution électronique Cairn.info pour Presses Universitaires de France.

(C) Presses Universitaires de France. Tous droits réservés pour tous pays.

La reproduction ou représentation de cet article, notamment par photocopie, n'est autorisée que dans les limites des conditions générales d'utilisation du site ou, le cas échéant, des conditions générales de la licence souscrite par votre établissement. Toute autre reproduction ou représentation, en tout ou partie, sous quelque forme et de quelque manière que ce soit, est interdite sauf accord préalable et écrit de l'éditeur, en dehors des cas prévus par la législation en vigueur en France. Il est précisé que son stockage dans une base de données est également interdit. 


\title{
Qui vient à Nuit Debout ? Paris, place de la République, avril-juin 2016 Trois méthodes pour une question
}

\section{Who comes to Nuit Debout? Paris, place de la République, April-June 2016 Three methods for a question}

\author{
par Stéphane Baciocchi ${ }^{*}$, Alexandra Bidet ${ }^{* *}$, Pierre Blavier ${ }^{* *}$, Carole Gayet- \\ Viaud $^{* * * *}$, Erwan Le Méner ${ }^{* * * *}$
}

$\mathbf{L}$ e 31 mars 2016 en fin d'après-midi, la place de la République est occupée dans la foulée d'une importante manifestation contre la « loi Travail ». Ce rassemblement inaugure Nuit Debout et, à Paris, une série d'autres rassemblements quotidiens, au même endroit, tout au long du printemps. La place ne regroupe pourtant pas seulement de farouches opposants à la loi El Khomri. Dès le premier jour, savoir qui vient sur la place fait même partie intégrante des activités qui s'y jouent et de la mobilisation qui s'y forme. Cette question fut en effet d'emblée au cœur des multiples hypothèses, tentatives de descriptions et disputes entre commentateurs, mais avant tout aussi entre participants. Comment, en conséquence, considérer toutes les formes de participation, depuis la simple présence passagère jusqu'aux formes d'implication les plus massives? Elles ne se répondent pas nécessairement, s'ignorent parfois, mais se donnent bien à observer, à décrire et à commenter, en premier lieu, pour les individus présents ensemble dans cet espace public. Nous avons donc cherché à tenir ensemble ces différentes formes de participation, tout en explorant la composition sociale du mouvement - pour pouvoir répondre à notre tour à la question : «Qui vient sur la place de la République? » En considérant qu'être présent sur la place valait comme participation à Nuit Debout, nous avons enquêté sur le rassemblement depuis son site même, en le saisissant dans son écologie et sa temporalité concrètes, plutôt qu'à travers sa seule genèse.

Une entrée attentive aux façons dont les pratiques s'inscrivent dans l'espace urbain et aux modes d'engagements concrets dans le rassemblement permet d'étudier son périmètre et les modalités d'engagement à partir des activités des passants, simples curieux, participants occasionnels, habitués, ou membres investis quotidiennement, jusqu'à devenir des piliers du mouvement. Nous avons, dans cette perspective, associé trois lignes d'investigation : 1) une analyse des caractéristiques d'un échantillon aléatoire de plus de 500 participants, ayant répondu à un questionnaire passé en face-à-face, in situ, entre avril et mai 2016 - pour certains des résultats : Collectif, 2017 - ; 2) un recensement intégral de la place mené le soir du 13 mai, associant dénombrement et relevé cartographique des présents ; 3) l'examen de questions ouvertes du questionnaire, en particulier «Selon vous, qui vient sur la place?»,

* Ingénieur de recherche (méthodes des sciences sociales) à l'Ehess

Ehess, $\mathrm{CRH}, 10$ rue Monsieur le Prince, 75006 Paris, France stephane.baciocchi@ehess.fr

* Chargée de recherche CNRS, Centre Maurice Halbwachs

$\mathrm{CMH}$, École normale supérieure/PSL, 48 boulevard Jourdan, 75014 Paris, France alexandra.bidet@ens.fr

*** Chargé de recherche CNRS, Centre lillois d'études et de recherches sociologiques et économiques Clersé, Bâtiment SH2 Cité Scientifique, Université de Lille, 59655 Villeneuve d'Ascq cedex, France pierre.blavier@univ-lille.fr

**** Chargée de recherche CNRS, Centre de recherches sociologiques sur le droit et les institutions pénales CESDIP, 43 boulevard Vauban, 78280 Guyancourt, France carole.gayetviaud@cesdip.fr 


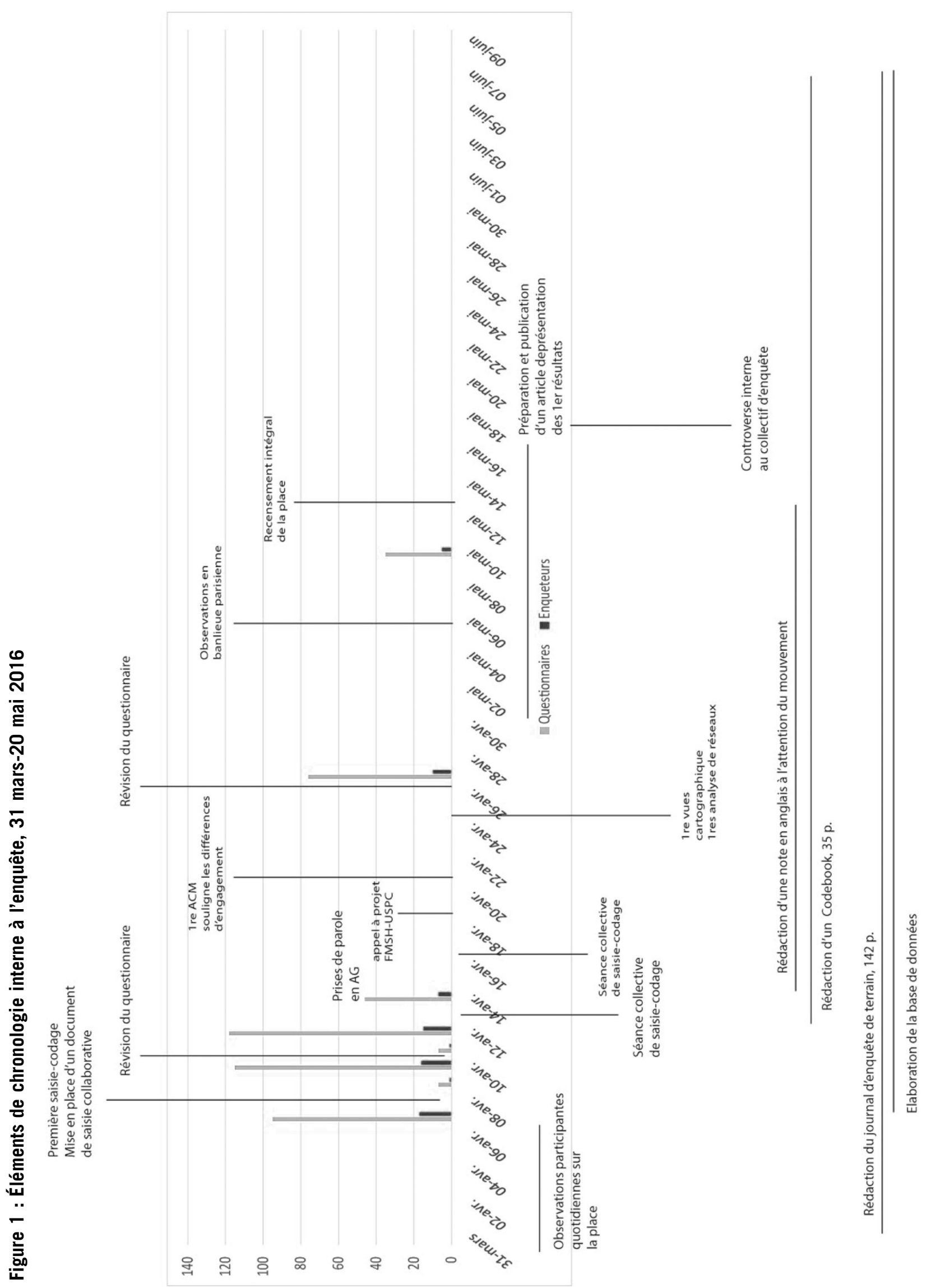


qu'ont ensuite complétées une trentaine d'entretiens approfondis auprès de participants.

Ces trois axes d'enquête ont été étroitement coordonnés. Si nous nous en étions tenus à interroger de façon non aléatoire les présents, comme l'ont fait de nombreux journalistes sur la place $^{1}$, nous n'aurions pu en former qu'une vue très partielle. Embrasser méthodiquement la diversité des personnes présentes, en couvrant toute la place et en tirant au sort les enquêtés, a permis de mieux interpréter les réponses à la question "Qui vient sur la place? », dont l'intérêt est étroitement lié à leur variété et à leur possible exhaustivité, lesquelles supposent bien sûr un certain effectif de personnes enquêtées. Ensuite, contrôler ce sondage, au sens statistique du terme, demandait une population de référence, donc une évaluation du nombre des présents sur la place et l'identification des formes de présence au sein de ce rassemblement, qui est aussi un ensemble de flux, un "lieu-mouvement » (Joseph, 1996), où se rencontre une pluralité de sous-espaces et d'ambiances. Enfin, les questions ouvertes et les entretiens ont permis de préciser le tableau d'ensemble en les associant à des perceptions situées de soi et des autres dans le mouvement, de ses contours, mais aussi à des dynamiques d'exploration et d'enquête internes au mouvement lui-même. Le souci d'établir «qui est là?» et, simultanément, « ce qu'est Nuit Debout », était omniprésent, non seulement chez les commentateurs, mais aussi parmi les participants, qui s'ouvraient parfois spontanément à nous de leur recherche inquiète ou passionnée d'un inventaire, d'un tri, voire d'une hiérarchie, entre des types de présences et de participation, des finalités, ou des orientations, plus "centrales » ou " originelles » de l'action collective : "Ce qui m'intéressait, c'était de savoir qui est-ce qu'il y avait... qui intervenait à Nuit Debout. J'ai pas réussi vraiment à identifier [...]. Est-ce que vous avez des réponses déjà? Quels sont les mecs récurrents, qui sont là, qui reviennent, qui se sont engagés dans les groupes? Pas les touristes comme moi, moi je suis un touriste si tu veux!» (C. $)^{2}$. Le séquençage temporel de ces trois lignes d'enquête est synthétisé dans une frise chronologique (Figure 1).
Le croisement de ces façons de se demander « qui vient sur la place » donne à comprendre Nuit Debout comme un espace hétérogène, accueillant une diversité de groupes, de présences, d'activités, d'enquêtes - comme le suggèrent les individus interrogés sur la place. En appréhendant de façon ample et ouverte le «qui » des participants, sans les hiérarchiser a priori selon des critères d'ancienneté, d'intensité, de visibilité ou de légitimité partisane, à la manière des participants et des commentateurs, on approche alors aussi le «quoi » et le « pourquoi »du mouvement, en rendant justice à la pluralité comme à la relative indétermination des motifs des présences et des contours du rassemblement. Sous le "quoi », on découvre des investissements fluctuant dans le temps, mais aussi des formes de participation et de présence plurielles, avec des centralités et des périphéries, des stations focalisées et des espaces de circulation, donc des co-présences variées. Elles invitent à penser différentes échelles de rassemblement, ainsi que la variété des regroupements et des façons de s'y engager.

\section{Passer un questionnaire et diffuser ses premiers résultats}

Enquêter in situ, pendant le mouvement, entre le 8 avril et le 13 mai 2016, a permis de décrire le rassemblement en cours, et non rétrospectivement comme le plus souvent ${ }^{3}$, tout en misant sur une familiarisation progressive à l'espace et à ses protagonistes, à même d'enrichir et de rendre plus pertinent notre questionnement. D'emblée portée par le projet de considérer simultanément tous les endroits de la place de la République, la plus grande place piétonne de Paris depuis sa réfection en 2013, l'enquête supposait un large groupe d'enquêteurs. La constitution impromptue d'un collectif d'enquête - 43 investigateurs au total ${ }^{4}$-, sans financement dédié ${ }^{5}$, par P. Blavier, qui débuta un journal de terrain dès le premier jour du rassemblement, le 31 mars, s'est faite à partir de trois viviers principaux : des amis parisiens, pour la plupart
1. Voir l'annexe électronique 1, https://journals.openedition.org/sociologie/ 7248.

2. Les lettres entre parenthèses renvoient aux enquêtés par questionnaire puis par entretien $(n=30)$. Les numéros entre parenthèses, plus bas dans le texte, correspondent à des portraits de participants réalisés par des journalistes, que nous avons rassemblés en corpus ( $n=130)$.
3. Voir par exemple (Occupy Research Network, 2012; Likki, 2012). A chaque fois les données proviennent de sondages réalisés ex post par internet et les potentiels biais de réponse sont très peu évoqués.

4. Voir le générique d'enquête en annexe électronique 4, https://journals.openedition.org/sociologie/7248.

5. L'exploitation de l'enquête a néanmoins bénéficié en 2016-2017 d'une subvention du Labex Tepsis (Ehess). 
universitaires, en sciences sociales, qu'il pensait pouvoir être intéressés par ce projet, des étudiants de l'ENS Cachan où il donnait depuis quelques années un cours d'initiation aux méthodes quantitatives en sciences sociales et des collègues doctorants ou enseignants-chercheurs ${ }^{6}$.

Chez les enquêteurs, les refus et quelques défections en cours de route se sont accompagnés de manifestations de réticence, ou de scepticisme parfois, à l'égard de l'idée même d'enquêter sur Nuit Debout; l'idéal, pour ces récalcitrants, était de rejoindre l'anonymat du simple participant, en émoussant la distance enquêteur/enquêté ${ }^{7}$ ou, plus sûrement encore, de s'engager autrement : dans et pour le mouvement. Ces retraits, souvent aiguisés par la hantise de « devenir les social scientists » ou "sociologues en chef» du mouvement, rejoignent des objections à l'enquête observées à l'extérieur de notre collectif d'enquêteurs, où le fait d'enquêter semblait être au risque, pour les uns, de pécher par excès de sympathie, et pour les autres, par excès de distance. Ainsi, un membre de la commission Lutte debout a pu appeler, lors d'un séminaire, à "ne pas voir Nuit Debout comme un objet d'étude ", et témoigner avoir cherché en vain à initier, sous l'intitulé " Cause commune et convergence des méthodes », une formule de recherche-action transversale au mouvement, " où tout le monde à la fin devient et chercheur et activiste ${ }^{8}$ ". De même, un organisateur nous rappela à la distinction entre « initiatives spectatrices » et "commissions actives » quand il découvrit dans un journal militant un article associant notre enquête à une commission Sciences sociales debout, «qui n'est pourtant pas référencée dans le wiki de Nuit Debout ». Des collègues se sont dits "gênés à l'idée de passer un questionnaire », compte tenu de ce qu'il implique comme distance à l'objet ; l'activité de recherche sur Nuit Debout semblait, plus généralement, devoir pour eux marquer une déférence, sinon une subordination, à l'égard de l'engagement militant ${ }^{9}$. Avoir une question de départ simple - «qui est sur la place? » - était donc sans doute décisif pour constituer un collectif d'enquête large et efficace; les énergies pouvaient converger vers un objectif concret et accessible, en même temps que chacun restait libre de lui associer des perspectives d'arrière-plan variées. Ainsi, l'initiateur de l'enquête pouvait avoir le souci de tester une analyse du mouvement en termes de précarité ${ }^{10}$, alors que d'autres s'intéressaient davantage aux trajectoires d'engagement des participants et à leurs différentes façons de faire l'expérience de Nuit Debout.

Le questionnaire, élaboré de façon itérative ${ }^{11}$, portait finalement à la fois sur les caractéristiques socio-démographiques des répondants, leurs parcours d'engagement et leurs formes d'activité sur la place et/ou dans le mouvement. Les enquêteurs étaient disposés à différents endroits, de sorte à couvrir la place aussi largement que possible. La consigne était de laisser passer neuf individus devant soi avant d'interpeller le dixième et de lui proposer de répondre au questionnaire ; les refus de participation étaient consignés. Le taux de refus de $14 \%$ est communément observé dans ce genre d'enquête en face-à-face sur le lieu même de la mobilisation politique (Walgrave \& Verhulst, 2011 ; Favre et al., 1997). II est frappant que les objections à l'enquête soient davantage venues des sciences sociales que de la place; alors que des commentateurs estimaient que I'humeur anti-institutionnelle de Nuit Debout motiverait de nombreux refus ${ }^{12}$, l'accueil du questionnaire fut particulièrement bon, y compris auprès de libertaires
6. Rencontrés pour la plupart dans le cadre du séminaire "Pratiques d'enquête et sens de la réalité sociale " d'A. Cottereau et S. Baciocchi, et liés pour certains par des pratiques d'enquête en commun, notamment dans le cadre du séminaire "Ethnographie des engagements » organisé, selon différentes formules, entre 2010 et 2016 par A. Bidet, M. Boutet, F. Chave, C. Gayet-Viaud et E. Le Méner.

7. Dans ses premières versions, le questionnaire se terminait par la proposition de "nous aider comme enquêteur » en contribuant lui aussi à faire passer des questionnaires.

8. Intervention relevée lors de la séance consacrée à Nuit Debout du séminaire " Pratiques utopiques » organisé par P. Sauvêtre et C. Laval à Nanterre le 10 mai 2016.

9. Lors du séminaire précédemment mentionné, les formes de présentation de soi de deux tiers des participants lors du tour de table manifestaient une recherche d'alignement sur un format militant. Pour la plupart universitaires, ils déclinaient, contrairement à ce qui est l'usage en séminaire, leur seul prénom, sans nom de famille, et parfois sans précision de statut, ou bien fournissaient des données mixtes, associant une inscription universitaire et une forme ou un lieu de participation à Nuit Debout.

10. Cette perspective, retraduite en termes de déclassement, sous-tend ainsi le premier article académique tiré de l'enquête (Collectif, 2017).

11. Voir l'annexe électronique 2, https://journals.openedition.org/sociologie/ 7248.

12. Dans un article de Politis faisant suite à la publication par dépêche AFP de nos premiers résultats partiels, Geoffrey Pleyers a ainsi estimé que, nombreux à Nuit Debout, "les libertaires, par exemple, qui refusent toute contrainte organisationnelle et institutionnelle ne répondront pas. [...] Je ne pense pas qu'on peut intégrer les milieux libertaires dans ce type d'enquête, notamment parce qu'ils occupent d'autres espaces et que la plupart sont très récalcitrants face à tous les questionnaires. » (M. Mazellier, "Qui sont les participants de Nuit Debout. Chercheurs et étudiants ont mené l'enquête place de la République. Le sociologue Geoffroy Pleyers analyse les résultats », Politis, 10 mai 2016) 
Figure 2 : Exemple de questionnaire, dans sa version finale
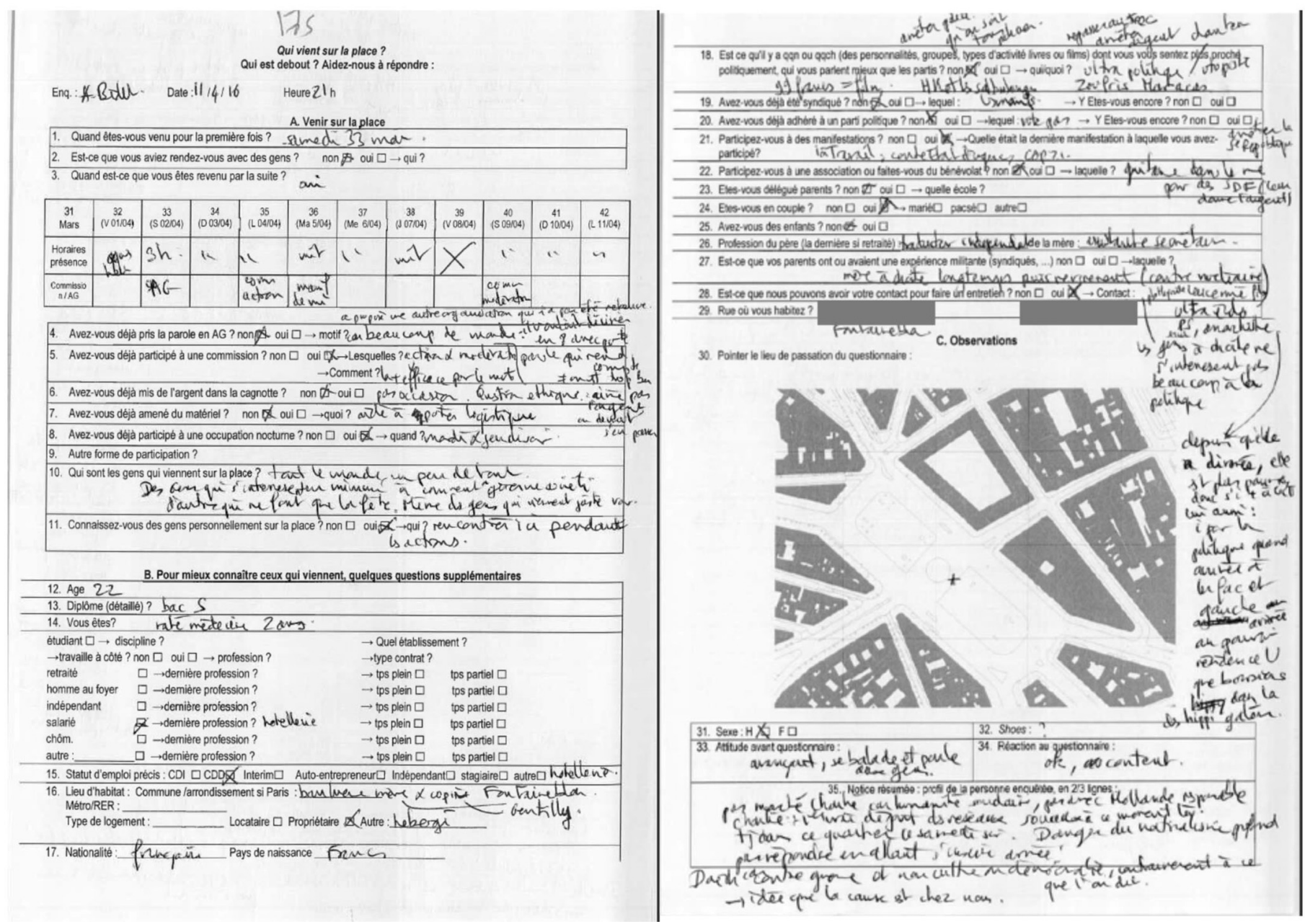

Questionnaire renseigné par l'enquêtrice A. Bidet le 11 avril 2016 à 21 heures, au pied (S-E) de la statue de la place de la République (Coll., Questionnaire 175, Archives de l'enquête "Qui vient sur la place? », conservées par P. Blavier).

et anarchistes, qui furent présents et enquêtés en nombre certains soirs. Les personnes semblaient plutôt percevoir l'enquête comme un prolongement de leurs propres questionnements et une occasion de contribuer à l'activité de la place, à travers une description mieux fondée de celle-ci. La passation du questionnaire, qui durait entre 15 et 45 minutes, se rapprochait alors d'une situation d'« entretien debout » : en face-à-face, avec prise de notes sous les yeux de l'enquêté, possible révision des propos en situation, et indexation des informations à des éléments environnants, directement observables $^{13}$. En outre, la présence de trois questions ouvertes, et les relances associées, conduisaient souvent à noircir les marges du questionnaire de notations (Figure 2) et à s'engager davantage dans l'interaction.

Au total, 514 questionnaires ont été ainsi passés en face-àface, à six dates différentes et à des horaires variés, entre 17 h 00 et 22 h 30 , par une dizaine d'enquêteurs à chaque fois, répartis de façon à couvrir toute la place - chaque questionnaire est associé à un endroit de la place -, et non pas seulement ses secteurs apparemment les plus impliqués, où se tenaient l'Assemblée générale, les stands et les réunions des commissions. Le réseau des enquêteurs peut être représenté par un graphe (voir Figure 3). 


\section{Figure 3 : Places et moments de la passation du questionnaire}

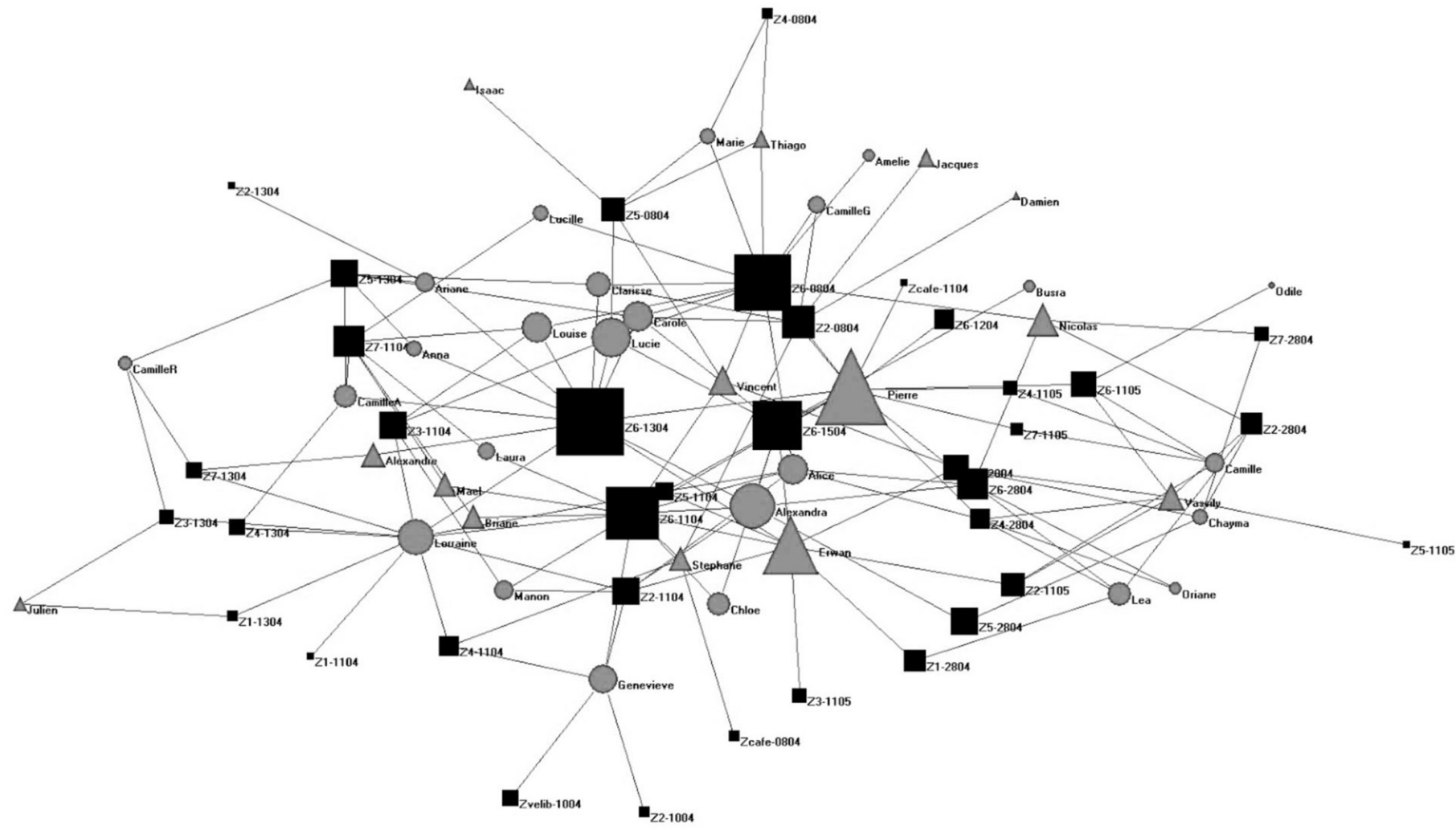

Lecture : les cercles et les triangles représentent les enquêteurs. Les femmes sont désignées par des cercles, les hommes par des triangles. Les carrés représentent les zones et personnes enquêtées (Z1 à Z7) aux différentes dates (« 1104 » désigne le 11 avril 2016). La taille des sommets est proportionnelle au nombre de questionnaires passés (notons que la distribution par sexe des enquêtés est la même que celle établie lors du recensement intégral). Au centre du graphique, où se trouve la plus grande densité de sommets, soit les principaux lieux et moments de l'enquête et leurs enquêteurs : la zone 6 est celle où se tient l'Assemblée générale et où les enquêteurs les plus actifs comme P. Blavier, E. Le Méner, A. Bidet et L. Champenois ont particulièrement travaillé les 8 , 11 , 13 et 15 avril 2016 . On note le travail de L. Guénée et E. Le Méner, sur des zones apparemment périphériques Z2, Z3, Z4... Les carrés montrent qu'il s'agit de groupes de personnes coprésentes, de groupes d'enquêtés plus (zone 6 le 9, 11, 1 avril et 15 avril) ou moins (Z7 ou Z2 le 28 avril, Z1 et Z3 le 11 ou 13 avril) importants.

Les passations et révisions du questionnaire se sont accompagnées, en cours d'enquête, d'un travail collectif d'exploitation concomitante des matériaux et de diffusion de premiers résultats. Sur la base des 328 premiers questionnaires, nous avons ainsi transmis une dépêche à l'AFP le 8 mai 2016, largement reprise le lendemain dans la presse nationale et régionale ; nous avons ensuite publié un article sous trois formats différents dans la presse généraliste mi-mai $2016^{14}$. II fut l'occasion de plaider pour une démarche d'enquête soucieuse d'établir des faits, à I'heure où les médias étaient saturés d'énoncés tranchants et non étayés, de catégories toutes faites et de hiérarchies a priori entre les endroits de la place, les moments ou les formes de participation, prétendant tous distinguer entre le «vrai » et le « faux » Nuit Debout. "La moyenne d'âge est de 25 ans », " un entre-soi de bobos parisiens », «aucun vrai prolétaire » mais " une bourgeoisie blanche urbaine », "des SDF et des punks à chien qui boivent de la bière », « un rassemblement d'étudiants déclassés, de militants de l'ultra-gauche et de semiprofessionnels de l'agit-prop » : ces énoncés, recensés parallèlement dans notre corpus d'articles de presse $(n=115)$, étaient autant de façons pour les commentateurs de valoriser ou dévaloriser ce qui s'y passait, d'estimer la valeur politique du mouvement, en clamant ce qu'il était, n'était pas ou devrait être. Aspirant à clore la question, ils s'interdisaient toute possibilité de
14. S. Baciocchi, A. Bidet, P. Blavier, M. Boutet, L. Champenois, C. GayetViaud, E. Le Méner, "Sortons des clichés à propos de Nuit Debout », Le Monde, Débats et analyses, 18 mai 2017, p. 21 ; "Nuit Debout est un rassemblement plus diversifié qu'on ne le dit», Le Monde.fr, 16 mai 2016, https://www.lemonde.fr/idees/article/2016/05/17/nuit-debout-est-un-rassem blement-plus-diversifie-qu-on-ne-le-dit 4920514 3232.html • "Qui vient à Nuit Debout? Des sociologues répondent », Reporterre, 17 mai 2016, https:// reporterre.net/Qui-vient-a-Nuit-debout-Des-sociologues-repondent. De premières interventions radio suivent : à Radio Debout, Radio Campus, Radio catholique française notamment. 


\section{Figure 4 : Année de naissance des personnes interrogées suivant l'heure de passation du questionnaire}

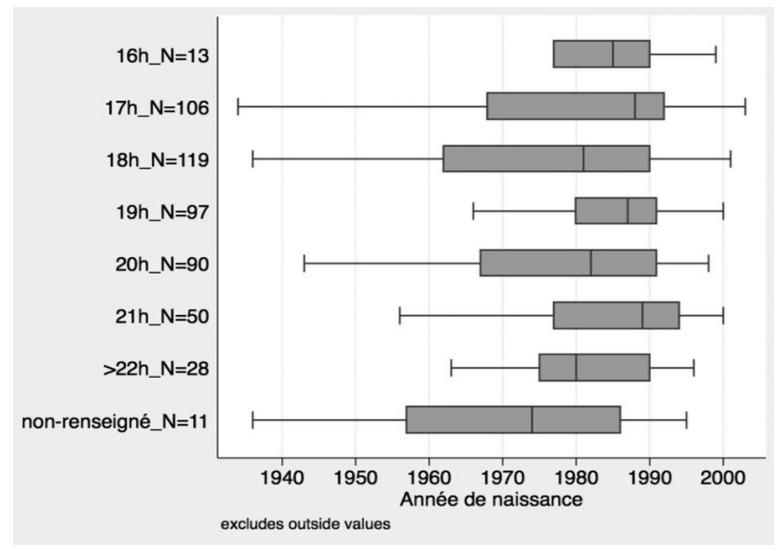

Champ : ensemble de l'échantillon, $N=514$. Lecture : la ligne au centre des boîtes, délimitées par les premiers et troisièmes quartiles, correspond à la médiane. Note : les effectifs par heure sont réduits, mais des tests de moyenne (dont l'hypothèse de normalité de la distribution par âge ne correspond pas bien à notre échantillon) montrent que certains écarts sont statistiquement significatifs.

surprise. Or, nos premiers résultats sur les gens « debout » au cours des premières semaines du mouvement montraient qu'il ne s'agissait pas simplement de jeunes, d'hommes, de Parisiens, de diplômés, d'une fête apolitique ou d'une foule amoureuse d'elle-même, comme on pouvait l'entendre alors dans les médias.

La palette des âges était par exemple très large et variait selon les heures. Autour de 18 heures, la moitié de la population avait plus de 33 ans et une personne sur cinq était âgée de plus de 50 ans (Figure 4).

De même, la population de la place n'est qu'aux deux tiers masculine $(60 \%)$ - un ratio retrouvé dans les autres Nuit Debout observées et, plus généralement, dans tous les mouvements de place, hormis le parc Gezi et la place Taksim à Istanbul (Konda, 2014) et le mouvement des parapluies à Hong Kong (Cheng \& Chan, 2016), où les enquêtes font état d'une parité. Du point de vue de l'origine résidentielle, les quartiers les plus représentés sont moins les quartiers centraux que ceux de l'Est parisien, comme le montre la carte des lieux de résidence des personnes présentes (Figure 5). Et si $37 \%$ des participants habitant en Île-de-France viennent en fait de banlieue, un participant sur dix n'habite même pas en région parisienne.

Que la majorité des participants soit diplômée du supérieur long (67,5 \% parmi les 25-49 ans) rejoint les constats dressés pour les autres mouvements de place, où les niveaux de diplômes sont systématiquement bien supérieurs à ceux de la population nationale. Dans le cas de Nuit Debout cependant, l'image se brouille (Collectif, 2017) : non seulement le taux de chômage est de $20 \%$ parmi les participants, soit le double de la moyenne nationale, mais on y dénombre $16 \%$ d'ouvriers parmi les actifs - trois fois plus qu'à Paris et autant que dans l'île-de-France prise dans son ensemble. En matière politique, plus d'un tiers des personnes a participé à une manifestation contre les réformes annoncées du Code du travail. La proportion des enquêtés déclarant avoir déjà été membre d'un parti politique est même remarquable dans un contexte de désaffection militante : $17 \%$. Et plus d'un sur cinq (22 \%) ont déjà cotisé à un syndicat. Les engagements citoyens, associatifs ou caritatifs sont également très représentés. Pour autant, la participation ne se réduit pas aux espaces et aux moments de débats, notamment autour de l'Assemblée générale : participer, ce peut être prendre part à distance en flânant le long des stands, ou encore diffuser auprès d'amis ou de collègues des mails, photos ou vidéos, venir parfois de loin, «pour regarder », se tenir au courant, ou pouvoir dire qu'on y a été, venir observer, explorer, voir si on peut être saisi par le mouvement, ou utile. Deux enquêtés sur trois ont apporté du matériel ou des denrées, donné de l'argent, pris la parole en assemblée générale ou participé à une commission. La participation active et assidue aux commissions - prise de notes, statut de « référent » - peut aussi se muer en un engagement à temps plein ; près de $10 \%$ des enquêtés sont ainsi devenus des quasi-permanents.

Les participants forment ainsi une population hétérogène et les formes de participation à Nuit Debout sont multiples. Le recensement des individus et des groupes présents sur la place nous a aidés à mieux saisir la circulation des participations et décrire les formes variées de coprésence. 
Figure 5 : Zones et foyers de recrutement des personnes présentes sur la place de la République durant le premier mois des rassemblements
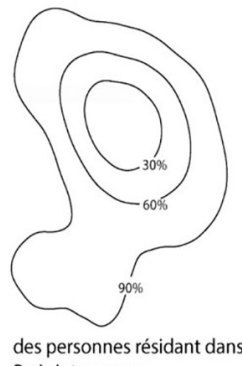

Paris intra-muros
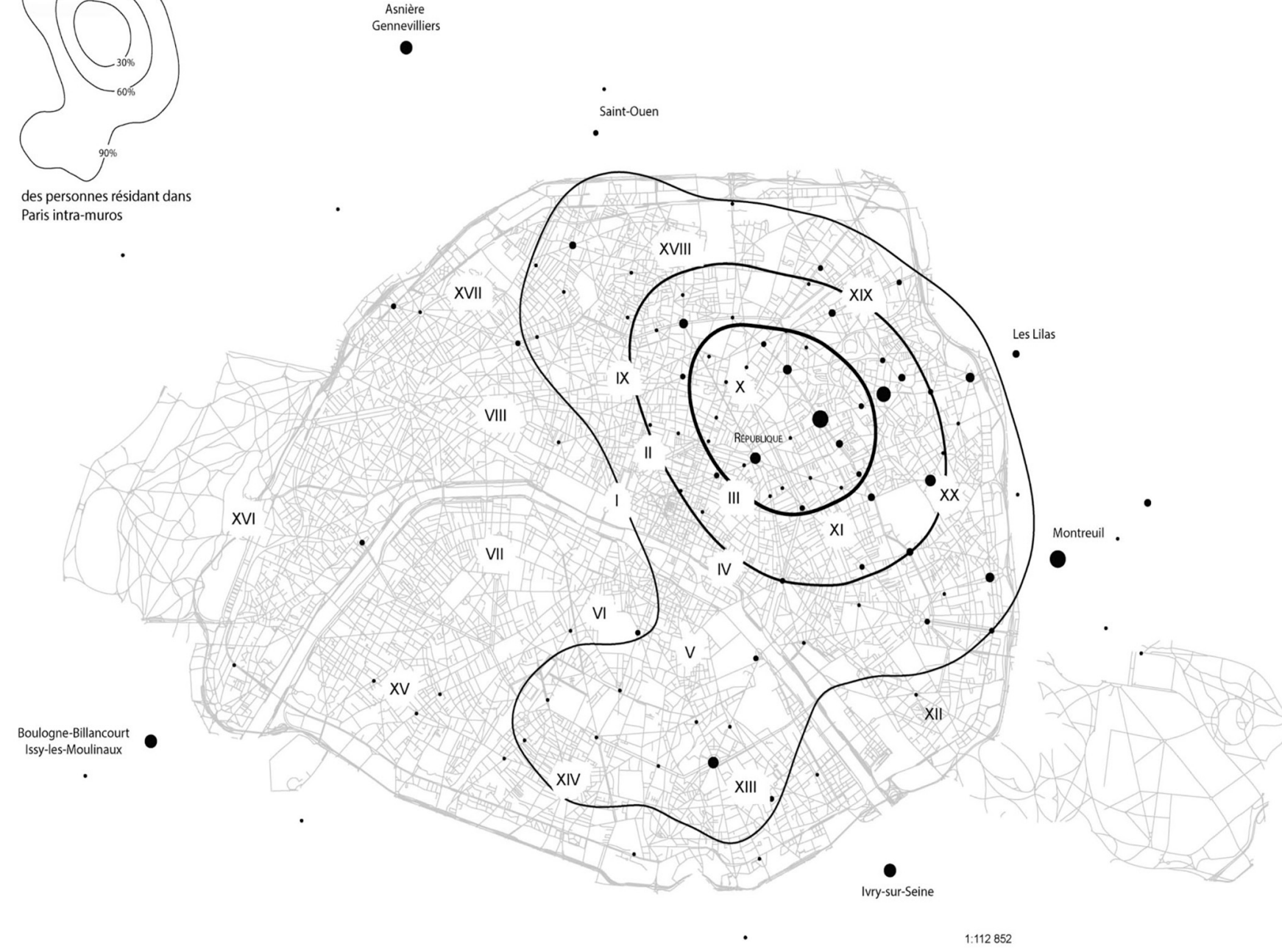

- Saint-Denis

Champ : présents habitant Paris et sa proche banlieue, $\mathrm{N}=359$. Lecture : les ellipses correspondent respectivement à 30/60/90 \% des personnes localisées selon la station de métro ou de RER la plus proche de leur domicile. Échelle 1:113000.

\section{Recenser les personnes présentes et cartographier les types de co-présences}

Le recensement intégral des personnes présentes sur la place ${ }^{15}$ a supposé son découpage en zones ${ }^{16}$, afin que chaque enquêteur puisse dénombrer et décrire les présents de manière systé- matique et coordonnée, en un temps limité, en minimisant les risques de doubles comptes ou d'oublis. Un plan et des itinéraires de recensement ont été établis en ce sens (voir Figure 6). Plusieurs passages ont ainsi été effectués au cours de la soirée, avec différents objectifs de repérage et de comptages (voir Tableau 1).
15. Effectué le 13 mai 2016, il a débuté à 19 h et s'est terminé à 23 h 30. L'attroupement avait parfois lieu plus tôt que $19 \mathrm{~h}$, en particulier les deux premières semaines, mais le 13 mai il était très réduit avant $18 \mathrm{~h}$. Le déroulé de la soirée a en revanche été similaire à celui observé auparavant.
16. L'est de la place a été divisé en quatre zones (4, 5, 6, 7), car la concentration de population y semblait plus élevée. 
Tableau 1 : Liste et objectifs des passages sur la place de la République, le 13 mai 2016

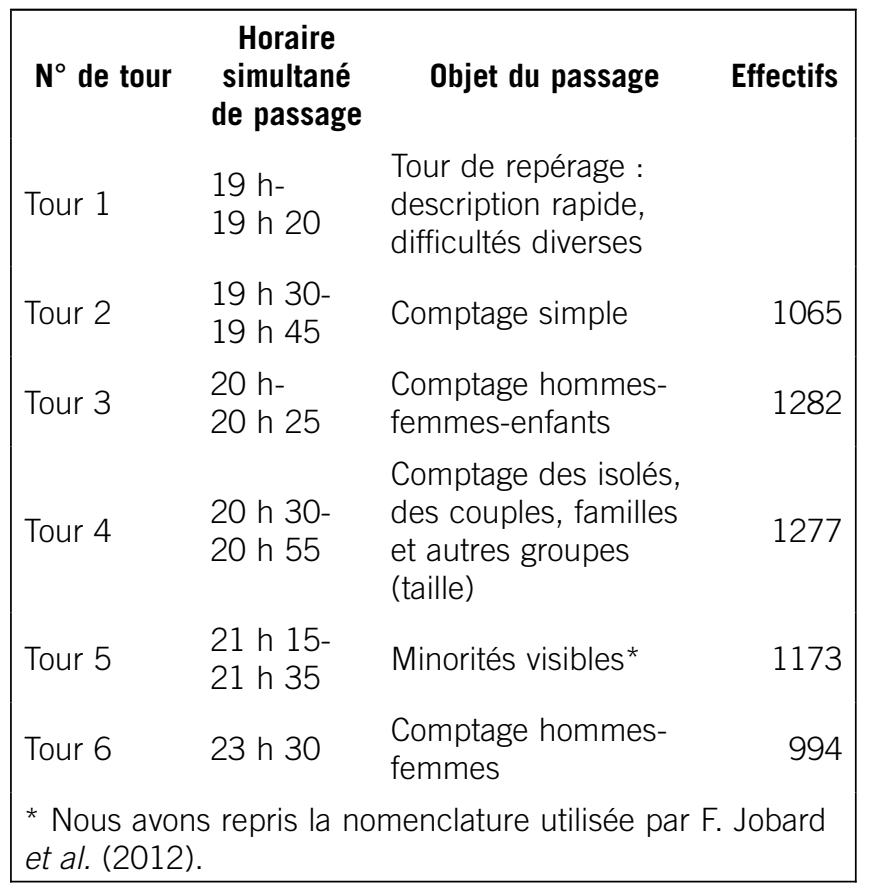

Ce recensement permet d'estimer le nombre de personnes présentes simultanément, qui oscille, à chaque heure, autour de 1 000. Avec notre échantillon de 514 questionnaires, nous avions donc un ratio de sondage connu et satisfaisant par rapport à la population de référence à l'instant $t$. Nous pouvions aussi caler l'échantillon sur les effectifs présents aux différents endroits de la place : la distribution spatiale des questionnaires s'avère en effet proche de la répartition dénombrée lors du recensement, même si nous avons sous-représenté la zone 2 du centre de la place, avec un fort taux de rotation, et sur-représenté la zone 6 où a lieu l'Assemblée générale, donc sur-pondéré la zone apparemment au cœur de ce rassemblement. Un biais vient aussi de l'absence, ce soirlà, de sound system, fréquemment présent à l'ouest de la place (zone 1) ; la projection d'un film après 22 h n'a pas attiré autant de monde. La répartition de notre échantillon sur la place est présentée dans le tableau 2.

Un autre résultat du recensement confirme nos observations et nos analyses des questionnaires. Le nombre de personnes présentes sur la place ne cesse de varier au fil de la soirée : 1065 personnes vers 19 h 30, lorsque nous commençons à compter, 1282 entre 20 h et 21 h, au plus fort de la soirée, puis 994 vers 23 h en fin de soirée. Enfin, l'effort pour situer l'échantillon a ouvert sur la nécessité de caractériser les différentes formes de coprésence et d'éléments qui coexistent sur la place. On a pu préciser les formes de regroupements : seulement un tiers des personnes sont isolées - i.e. venues apparemment seules. Plusieurs indices ont permis d'identifier des groupes ou des duos : des postures - être proches, se tenir la main, être en cercle, etc. -, des échanges de paroles ou d'objets - vêtements, bouteilles, stylos, etc. L'Assemblée générale est le seul endroit où ces critères se sont avérés inopérants pour repérer des formes de liens moins faibles que ceux de la simple coprésence en «public » (Joseph, 2003). Nous présentons sur la figure 7 un extrait d'un cahier de recensement de la partie nord-ouest de la place, sur lequel ont été figurées ex post les densités.

L'étude comparée des densités dans les différentes régions de la place incite aussi à reconsidérer sa morphologie sociale. Loin de l'opposition, par exemple, entre une partie ouest de la place qui «rit, danse, boit et s'ébat » à une partie est, « plus

\section{Tableau 2 : Répartition de l'échantillon sur la place de la République, le 13 mai 2016}

\begin{tabular}{|lccccc|}
\hline $\begin{array}{l}\text { Zones } \\
\text { (Figure 6) }\end{array}$ & $\begin{array}{c}\text { Nombre d'individus } \\
\text { recensés sur la soirée }\end{array}$ & $\begin{array}{c}\text { Part dans le } \\
\text { recensement }\end{array}$ & $\begin{array}{c}\text { Effectif dans les } \\
\text { questionnaires }\end{array}$ & $\begin{array}{c}\text { Part dans les } \\
\text { questionnaires }\end{array}$ & $\begin{array}{c}\text { Écart (recensement } \\
\text { moins questionnaires) }\end{array}$ \\
Zone 1 & 229 & $3,9 \%$ & 15 & $3,2 \%$ & $0,7 \%$ \\
Zone 2 & 1710 & $29,5 \%$ & 65 & $14,1 \%$ & $\mathbf{1 5 , 4} \%$ \\
Zone 3 & 281 & $4,8 \%$ & 37 & $8,0 \%$ & $-3,2 \%$ \\
Zone 4 & 388 & $6,7 \%$ & 20 & $4,3 \%$ & $2,4 \%$ \\
Zone 5 & 791 & $13,6 \%$ & 44 & $9,5 \%$ & $4,1 \%$ \\
Zone 6 & 1863 & $32,2 \%$ & 261 & $56,5 \%$ & $\mathbf{- 2 4 , 3 \%}$ \\
Zone 7 & 529 & $9,1 \%$ & 20 & $4,3 \%$ & $4,8 \%$ \\
Autre (non renseigné) & & & 52 & $11,3 \%$ & $100 \%$ \\
TOTAL & 5791 & $100 \%$ & 462 & \\
\hline
\end{tabular}


Figure 6 : Plan et itinéraires de circulation des enquêteurs pour réaliser de manière coordonnée et exhaustive le comptage de visu des personnes présentes sur la place de la République

Les numéros sont ceux des 7 zones de recensement, les points sont les arbres d'alignement, les étoiles les candélabres. La place est délimitée par un trait continu, tout comme le contour de la statue, l'emprise du bar sur la place, les limites des plateformes et les passages piétons qui donnent accès à la place

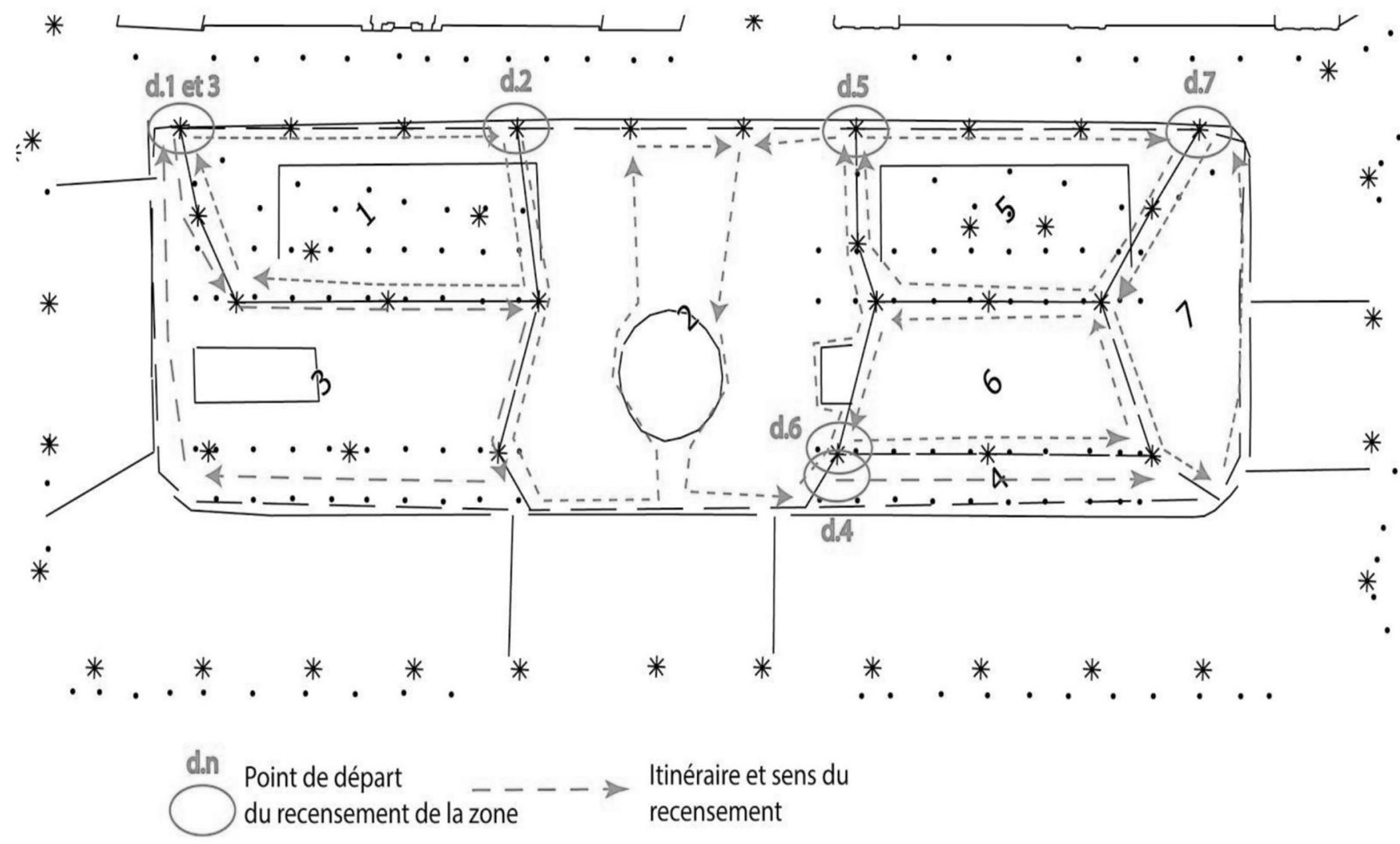

Extrait de S. Baciocchi, Recenser la place de la République. Petit guide de l'enquêteur, 13 mai 2016, 10 p.

éduquée », "qui pense » et " débat ${ }^{17}$ ", on peut distinguer plusieurs formes de coprésence et échelles de rassemblement, et leurs entrelacements sur la place.

Si l'on considère la carte globale de densité et des stands de la place de la République (Figure 8), on voit qu'à côté du rassemblement central de l'Assemblée générale, orienté vers la tribune, se déploient de multiples rassemblements focalisés, de différentes tailles, mais aussi des formes de coprésence davantage associées à des rassemblements d'une à trois personnes. Sur la figure 9 le graphique de droite représente les rassemblements focalisés, correspondant essentiellement au public de l'Assemblée générale et à celui concentré par les stands. Ailleurs, en dehors des marches et du cinéma en plein air, l'activité est nettement plus diffuse et les concentrations moindres. Cette hétérogénéité du rassemblement, des formes de présence et de participation déployées sur la place, amène à en tracer autrement les " contours ». En termes écologiques, il ne s'agit plus d'un partage est/ouest, mais d'une nappe dentelée de rassemblements qui représente la contiguiité spatiale des participants du rassemblement (Figure 8). À l'intérieur de ces contours, les personnes sont en effet à moins de deux mètres de distance (Figure 9). 


\section{Figure 7 : Pointage des personnes présentes dans les zones de recensement 1 et 3 entre $x x h . x x$ et $x x h . x x$}

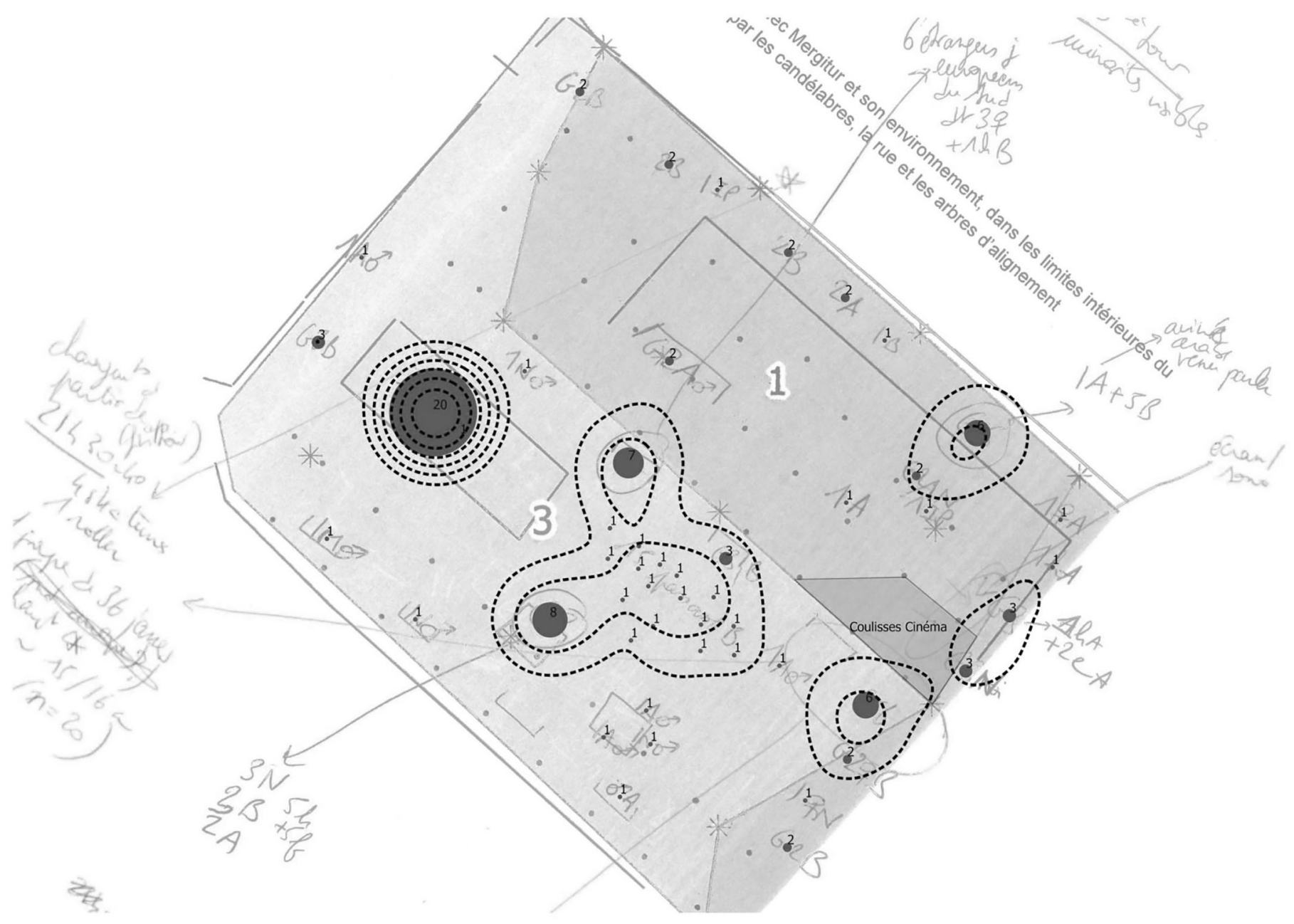

Les comptages réalisés par les enquêteurs et directement portés sur le plan (marques manuscrites) ont été vectorisés et analysés comme densités spatiales (Collectif, Carton « Recensement du 13 mai », Archives de l'enquête "Qui vient sur la place? », conservées par P. Blavier).

Nuit Debout, malgré ses niches et ses recoins, apparaît donc bien comme un milieu où la contiguïté, l'accessibilité et l'exposition à autrui sont fortes. Si la distribution des présences montre que peu de personnes ou de groupes sont isolés dans une zone, l'attention aux circulations met en évidence de nombreuses jonctions entre les espaces et les rassemblements repérés sur la place. La large nappe centrale est en effet aussi un espace de communication vers l'est et l'ouest, sur lesquels elle empiète ; et, autour de la tribune, s'observe un espace de circulation en U reliant le stand « grève générale » à la cantine.

Cartographier ainsi les présences constitue une entrée inédite pour décrire les mouvements de place, et pénétrer ici le jeu, relevé par Véronique Nahoum-Grappe (2016, p. 145-146), entre la "scène affairée » et relativement stable de Nuit Debout et la configuration mouvante de l'ensemble. Mais répondre à la question "qui vient sur la place » suppose aussi de considérer les perceptions situées de cet environnement par les participants eux-mêmes. Exploiter la question ouverte «selon vous, qui sont les gens qui viennent sur la place? » a ainsi permis de creuser la perspective écologique adoptée : envisager la place de la République dans son entier, non pas comme une simple surface ou une enveloppe, mais comme un environnement pour ses participants, dont la forme, l'équipement, les plis et les niches configurent les activités. 
Figure 8 : Personnes présentes sur la place de la République le soir du recensement

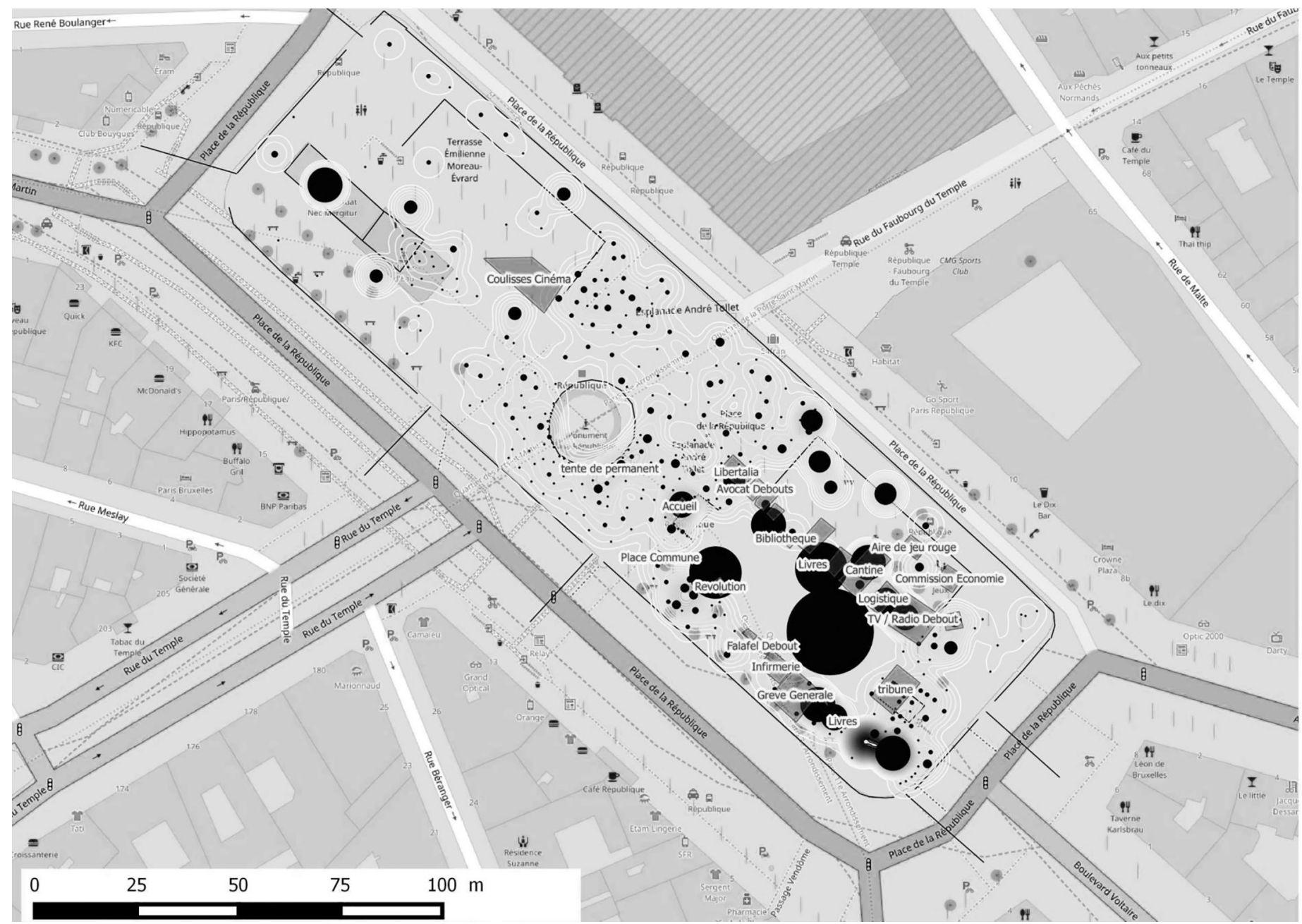

Figure 9 : Les personnes présentes sur la place de la République le 13 mai 2016
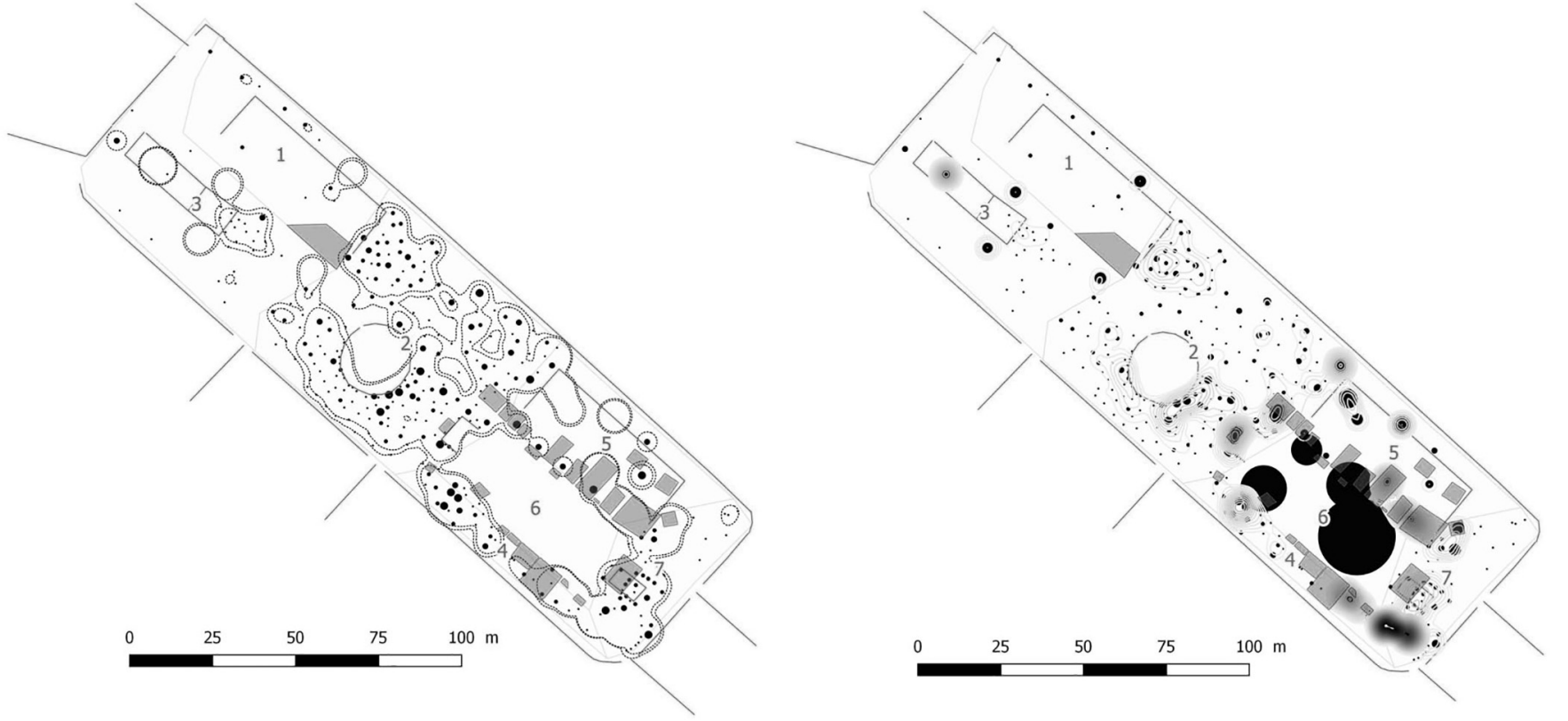


\section{Exploiter une question ouverte : "Selon vous, qui sont les gens qui viennent sur la place? »}

Les participants livrent une foule d'énoncés, qui sont autant de fragments pertinents, au sens d'Alfred Schütz (1970) ${ }^{18}$, d'une description de la place par les gens qui s'y trouvent assemblés. Elles permettent d'explorer les « catégories ordinaires de repérage » du monde social (Boltanski \& Thévenot, 2015 [1983]) ici mises en œuvre dans un exercice pratique d'orientation, indissociable d'une écologie plus générale de la perception.

"C'est varié », "C'est divers » : si 21 \% des 514 personnes interrogées décrivent la place en ces termes, elles s'arrêtent rarement là. Le propre du "divers »(Mauss, 1936, p. 6) est qu'il ne peut pas être énoncé en une phrase synthétique, au-delà du terme même, communément utilisé pour qualifier ce qui demeure incompris : il appelle l'inventaire, le classement. Or, dans le cas de Nuit Debout, nous allons voir qu'il n'est pas non plus infini. Les enquêtés notent la variété des personnes présentes (21\%), ou bien la présence de tout le monde ou presque $(11 \%)$. Selon la première modalité de réponse, Nuit Debout "brasse large». Deux perceptions dominent : il y a «de tout» $(n=56)$ ou " un peu de tout» ( $n=28$ ) sur la place, " un mouvement pluriel », "plein de gens différents » $(n=6)$, une grande "diversité »- le mot et des dérivés lexicaux sont utilisés à 22 reprises. Cette « hétérogénéité » $(n=10)$ n'empêche pas des caractérisations plus fines, ni le constat de variations temporelles. Le caractère " extrêmement diversifié », " mélangé » $(n=10)$ ou " varié » $(n=25)$ - selon les termes les plus cités - du rassemblement apparaît ainsi, aux participants comme à l'observateur, plus important le week-end. Ces personnes de "toutes sortes", "tous horizons", ces gens de «tout type», de "tous genres", ces «groupes» "différents», composent un ensemble " hétérogène », sinon « hétéroclite ». Cette "population extraordinairement diverse » peut être valorisée comme une « richesse », une "force » et le rassemblement, associé à une joyeuse "kermesse » $(n=4)$, avec une « bonne cohabitation de tous les groupes sociaux».

La deuxième modalité de réponse, souvent très proche, perçoit un «tout » $(n=86)$ sur la place. II peut s'agir d'un tout indifférencié, sinon indifférent : " tout le monde et personne », « pas d'opinion » ou, au contraire, d'une diversité. "Tout le monde», "presque tout le monde», "un peu tout le monde ", ce peut être aussi la figure unitaire, sinon solidaire du «peuple» : "le peuple dans son intégralité, on est tous dans le même cas»; ou rassemblé sur un «lieu identifié comme un espace de réunion », dans un "rassemblement de discussions ». "Tout le monde », ce peut être aussi « toute la France » ou «toute la population». Parfois, cette totalité n'est qu'à l'horizon : «on aura besoin de tout le monde »; de « tout bon citoyen, ça doit transcender toutes les appartenances sociales "; "y'a beaucoup de gens qui sont pas là, mais qui seraient bien là. Je connais beaucoup de gens qui aimeraient être là. II faut avoir les moyens de venir ». La totalité peut aussi renvoyer à une diversité sociale ou culturelle : «il y a de tout », " un peu de tout», "la société française, francilienne, dans sa diversité », « un peu tout le monde multiculturel», «pas une classe sociale qui ressort». Ce côté «varié » se trouve valorisé, soit qu'il attire : "ici il y a tout le monde, c'est pour ça que je viens »; soit que les absents soient des repoussoirs : " de tout à part les fachos »; soit qu'il engage une représentativité du mouvement : «99\% de la population », " tout est représenté, pas forcément en proportions égales ».

Percevant une diversité de gens, les participants saisissent simultanément la place comme "tout » : au fond, estime un enquêté, "à la hauteur ou pas intellectuellement, [les gens sur la place] participent à un ensemble ». Cet ensemble peut renvoyer à la citoyenneté, quand sont perçus des citoyens français de tous horizons, "conscients ", "actifs ", "positifs », qui " se sentent investis d'une mission démocratique ». Mais il englobe surtout de nombreux "curieux », évoqués par $8 \%$ des répondants, qui voient sur la place toute une population de passage : "tout le monde, un peu tout le monde, des gens qui viennent juste voir », "beaucoup de curieux», "beaucoup d'observateurs", «beaucoup de passants", "beaucoup de touristes", "des gens qui passent », "qui viennent découvrir », " juste là pour voir ».

Ces vues d'ensemble dessinent aussi des polarisations : « il y a des gens qui passent, d'autres qui s'engagent », des " gens engagés ou juste là pour voir ». Un observateur propose même 
une estimation : "curieux $50 \%$, engagés $50 \%$ ». Une autre polarisation renvoie au côté «festif » de la place, qui n'est toutefois thématisé expressément que par $2 \%$ des répondants et ne les conduit jamais à réduire la place à un festival, fût-il politique ${ }^{19}$. Ils voient "des gens qui font de la musique », "d'autres qui ne font que la fête » ou « se divertir » et « des gens des festivals ». Tout en s'exclamant «c'est la kermesse ici ! Ça me fait marrer! », un enquêté "sent qu'il y a quelque chose de sérieux et les gens viennent en conséquence ». Audelà de cette hybridation, la qualité globale du rassemblement, comme environnement perçu, « hypnotique » (63), « jamais vu » (8), « inédit » (58) ou encore "fantastique » (79), en fait aussi la singularité pour ses participants : « quand je sors d'ici, le monde me paraît ennuyeux. On vit un truc indescriptible » (19) 20

Les « abords » de Nuit Debout ne sont donc ni ses marges ni ses à-côtés. L'étude des énoncés rejoint le recensement et nos observations : non seulement ces abords sont partie prenante du rassemblement, comme environnement perçu des participants, dont plus de $80 \%$ viennent de façon occasionnelle, mais ils s'intègrent aussi aux circulations. Des participants identifient et valorisent l'originalité du mouvement : " c'est très différent des manifs habituelles, où tout le monde avance ensemble, mais où personne ne se parle. On a enfin un vrai espace où l'on entend des vrais gens parler » (50), " on se parle!»; «on se reparle enfin » (4) ; « ici, les gens peuvent simplement passer, échanger [...] aujourd'hui en démocratie, nous votons mais nous ne disons rien » (122). II s'agit peutêtre moins de penser que de déambuler dans « un endroit où ça bouge, ça circule, ça discute, ça rêve, ça palpite » (87). On a beaucoup parlé «d'entre-soi » à propos de Nuit Debout, sans jamais préciser ce terme, ni observer que la place, au fil de ses différentes configurations, abritait en fait une pluralité d'entre-soi dans ses niches ou recoins.

Les deux allées de stands qui bordent l'assemblée générale sur toute sa longueur, tel un mur d'enceintes et d'écho, évoque à certains « un petit village rempli d'expériences nou- velles » ou un « laboratoire d'expériences politiques ». En arrivant, certains commencent ainsi par déambuler dans ces allées : " J'y suis allée un peu en observation, la première fois, juste pour me promener dans les allées, ils étaient en train de monter les stands, donc c'était intéressant. » La figure paradoxale du village urbain dit l'hospitalité aux « essais » et aux initiatives : «ici tout le monde est ouvert, on peut décider de faire une action, de monter une tente, de gérer un endroit comme les poubelles, et tout le monde est d'accord »; «tu viens pour faire ce qu'il te plaît »; « ici on est vraiment libres ». Le contact se fait très aisément. Aussitôt arrivé, J. est comme happé par le rassemblement : "Je me suis retrouvé avec quatre mecs que je ne connaissais ni d'Ève ni d'Adam à essayer d'arracher des clôtures de chantier [...], au bout de deux jours, j'étais chez moi, je tutoyais tout le monde, je prenais le numéro de téléphone de tout le monde [...] et je me baladais comme si j'étais chez moi sur la place» (J.). Un autre enquêté (A.) narre maintes situations où, alors qu'il discutait avec des inconnus, on a cru qu'il était avec des parents.

Le format « inédit » de ce rassemblement vaut pour certains à lui seul : «Moi je prends mon pied quand je viens ici [...], j'aimais cette ambiance de gens qui discutent entre eux, voilà, c'est déjà bien de discuter [...] et puis, surtout, ce que j'ai apprécié, c'est l'extrême respect que les gens essayaient de manifester vis-à-vis de toutes les interventions. Y'avait du respect quand même dans la parole, ce que nous on n'avait pas à l'époque en 1968. [...] Là [à Nuit Debout], les gens trouvaient intéressant de parler, même si la discussion était pas intéressante» (C.) ; «ce que j'ai aimé, c'est la bienveillance des prises de parole. Un profond respect pour la parole des gens [...] Y'a une volonté inclusive évidente »(Lt.). Le pouvoir d'attraction du rassemblement semble souvent irrépressible : «je n'arrive pas à m'empêcher de passer chaque jour trois quatre heures », " je n'ai pas pu m'empêcher », " je viens rêver tous les jours", "je voudrais qu'il y ait tout le temps du monde ici, qu'il y ait une assemblée générale permanente », etc. ${ }^{21}$ Rêver, mais aussi explorer : la posture
19. L'expression de "festival politique », employée par P. Ngo et C. Truong (2016, p. 12), semble ignorer la tension sous-jacente, dont témoigne aussi notre corpus d'interviews et d'entretiens, notamment quand sont évoqués le bruit des animations sonores, qui empêche parfois de discuter ou de bien suivre les débats comme s'en agace/offusque Étienne Brunet (http:// www.etiennebru.net/une_petite_musique_debout.pdf, pages 7, 14-15, 16, etc., consulté le $1^{\text {er }}$ décembre 2016) ou le problème des vendeurs à la sauvette, qui « introduisent l'argent sur la place » (P.).
20. Sur le rôle à cet égard de la dimension nocturne du rassemblement, voir (Bidet et al., à paraître).

21. Voir aussi en ce sens le témoignage de Constance (Ngo \& Truong, 2016, p. 31). 
d'enquête est fréquente. Nombreux sont ainsi ceux qui ont observé la " librairie en libre-service », à la manière d'un test pour leurs croyances et le mouvement lui-même : "c'était intéressant de savoir si... on a juste discuté de savoir comment ça fonctionnait, si ça fonctionnait bien, si les gens en effet donnaient des livres, s'il n'y avait pas juste des gens qui prenaient et personne qui ne donnait [...] ben non, ça a l'air de bien fonctionner, donc c'est cool, ça redonne un peu espoir en l'être humain » (L.). De même, pour la «cantine libre» située à proximité : "Je trouvais cela génial, parce que les gens... Enfin, quand on dit "oui, c'est prix libre, les gens vont rien donner", mais finalement, ils donnent. Nous, on le voit à Nuit Debout Lilas, on a fait un petit apéro, et les gens prennent, mais même s'ils donnent vingt centimes ou quoi, ils donnent quand même. Et ça je pense que c'était quand même intéressant » (Je.).

\section{Conclusion : un espace d'enquêtes sur le politique}

Parce qu'un rassemblement n'est pas un groupe dont on analyserait la sociabilité dans les formes du "nous », les différentes façons de dire l'accord et d'afficher sa compétence doivent être « redonnées », reprises comme de nouveaux observables (Joseph, 1996, p. 25).

Des statistiques à l'analyse d'énoncés, en passant par l'opération de recensement, nos trois façons de répondre à la question « qui vient sur la place? » à Nuit Debout ouvrent des perspectives inédites sur la nature et les motifs de ce rassemblement de place, le « quoi » et le « pourquoi » du mouvement.

L'enquête inscrit sa démarche dans la lignée des réflexions aujourd'hui foisonnantes sur les liens entre ville et mouvements sociaux. Si les places publiques ont tendance à voir un réinvestissement citoyen de la chose publique, leur politisation a du moins connu un regain de faveur global à l'occasion des mouvements d'occupation, ces «mouvements de places», dans la lignée desquels Nuit Debout s'est pour partie situé. À la différence de tels rassemblements, l'occupation n'a pas pu durer, en raison notamment de la morphologie de la place
(Zask, 2018). Toutefois, il nous semble que la forme de cet espace, sous l'aspect des engagements multiples qui s'y sont déployés, a pu favoriser un autre genre de rassemblement. À certains égards, le mouvement est ainsi apparu plus proche du festival ${ }^{22}$, ou de la foire aux idées politiques, que de la manifestation ou de l'occupation. II a offert une conjonction singulière de rapports distants et anonymes, propres à la sociabilité urbaine ordinaire, et d'engagements focalisés nourris d'une présomption de politisation, propres aux espaces de débats. Ces formes de côtoiement et d'échange tranchaient avec l'urbanité alentour, tout en se voulant ouvertes au toutvenant : l'accessibilité et la porosité étaient envisagées à la fois comme des moyens et des fins de l'effort collectif d'organisation et d'expérimentation.

Intégrer à l'analyse la morphologie du rassemblement et la variété de ses "degrés de conviction », allant des attachements fugaces aux élans prosélytes, nous renseigne sur l'originalité qui se cherche à travers les mouvements de place contemporains. Ils rapprochent, dans le même espace-temps, deux formes qui étaient plutôt alternées au sein des apprentissages politiques au $X^{\mathrm{e}}$ siècle - nocturnes et diurnes, café et manifestation. Ce que nous avons été amenés à décrire n'est pas en effet seulement une co-extension de ces formes, mais leur tissage fin : à des endroits, la trame est serrée et permet la continuité d'un «travail »; à d'autres, elle est suffisamment relâchée pour laisser, au gré des explorations et des occasions, naître et s'éteindre mille conversations. L'ensemble forme ainsi une nappe, ou une couverture assez lâche de la place, qui en fait un espace propice aux circulations, c'est-à-dire finalement à une modulation individuelle des engagements personnels tout au long d'une soirée, qui offre d'une façon inédite à chacun la possibilité d'expérimenter son attachement aux idées avancées aussi bien qu'au mouvement en général. La question, souvent posée dans les médias, des "débouchés » politiques du mouvement ne peut alors plus être réduite à sa dimension partisane ; elle appelle à documenter, bien plus largement, les effets des multiples expériences et enquêtes menées par les participants sur la place - et de leurs façons d'y localiser ce qui vaut comme «politique ». 


\section{Bibliographie}

Bidet A., Le Méner E., Baciocchi S., Blavier P., Boutet M. \& Gayet-Viaud C. (à paraître), "Les nuits de Nuit Debout : conflits et expériences autour d'un temps ouvert », in Garnier G. \& Ambroise-Rendu A.-C. (dir.), La Nuit blanche de l'Antiquité à nos jours.

Boltanski L. \& Thévenot L. (2015 [1983]), «Comment s'orienter dans le monde social », Sociologie, vol. 6, n 1, p. 5-30 [traduction de "Finding One's Way in Social Space: A Study Based On Games », 1983].

Cheng E. W. \& Chan W.-Y. (2016), « Explaining Spontaneous Occupation: Antecedents, Contingencies and Spaces in the Umbrella Movement », Social Movement Studies, vol. 16, n 2 , p. 222-239.

Collectif (2017), "Déclassement sectoriel et rassemblement public. Éléments de sociographie de Nuit Debout place de la République ", Revue française de science politique, vol. 67, $n^{\circ} 4$, p. 675-693.

Favre P., Fillieule 0. \& Mayer N. (1997), « La fin d'une étrange lacune de la sociologie des mobilisations. L'étude par sondage des manifestants. Fondements théoriques et solutions techniques ", Revue française de science politique, vol. 47, $\mathrm{n}^{\circ} 1$, p. 3-28.

Jobard F., Lévy R., Lamberth J. \& Névanen S. (2012), « Mesurer les discriminations selon l'apparence et les contrôles d'identité à Paris », Population, vol. 67, n 3, p. 423-451.
Joseph I. (1996), "Les compétences de rassemblement», Enquête, no 4, p. 107-122.

Joseph I. (2003), «La notion de public : Simmel, l'écologie urbaine et Goffman », in Cefaï D. \& Pasquier D. (dir.), Les Sens du public. Publics politiques, publics médiatiques, Paris, Puf. Konda (2014), Gezi Report, June, https://konda.com.tr/wpcontent/uploads/2017/03/KONDA_Gezi_Report.pdf (consulté le 26 mars 2020).

Likki T. (2012), «15M Revisited: A Diverse Movement United for Change », Zoom Político, no 11.

Mauss M. (1936), "Les techniques du corps », Journal de psychologie, vol. 32, n० 3-4, p. 271-293.

Nahoum-Grappe V. (2016), "Nuit Debout. Place de la République. 31-47 mars 2016 », Esprit, n 7, p. 145-146.

Ngo Ph. \& Truong C. (2016), Nuit(s) debout, Paris, Atlande.

Occupy Research Network (2012), "Preliminary Findings: Occupy Research Demographic and Political Participation Survey ".

Schütz A. (1971), Reflections in the Problem of Relevance, edited by R. M. Zaner, New Haven, Yale University Press.

Walgrave S. \& Verhulst J. (2011), "Selection and Response Bias in Protest Surveys", Mobilization: An International Journal, vol. 16, n 2, p. 203-222.

Zask J. (2018), Quand la place devient publique, Paris, Le Bord de l'eau. 